\title{
NEW SUBCLASS OF UNIVALENT FUNCTIONS DEFINED BY USING GENERALIZED SALAGEAN OPERATOR
}

\author{
S. B. Joshi And N. D. SAngle
}

\begin{abstract}
In this paper, we have introduced and studied a new subclass $T D_{\lambda}(\alpha, \beta, \xi ; n)$ of univalent functions defined by using generalized Salagean operator in the unit disk $U=\{z:|z|<1\}$ We have obtained among others results like, coefficient inequalities, distortion theorem, extreme points, neighbourhood and Hadamard product properties.
\end{abstract}

\section{INTRODUCTION}

Let $A$ denote the class of functions of the form

$$
f(z)=z+\sum_{k=2}^{\infty} a_{k} z^{k}
$$

which are analytic in the unit disk $U=\{z:|z|<1\}$. In [4], Al-oboudi defined a differential operator as follows, for a function $f \in A$,

$$
\begin{aligned}
D^{0} f(z) & =f(z), \\
D f(z) & =D^{1} f(z)=(1-\lambda) f(z)+\lambda z f^{\prime}(z)=D_{\lambda} f(z), \lambda \geq 0,
\end{aligned}
$$

in general

$$
D^{n} f(z)=D_{\lambda}\left(D^{n-1} f(z)\right) .
$$

If $f(z)$ is given by (1), then from (2) and (3) we observe that

$$
D^{n} f(z)=z+\sum_{k=2}^{\infty}[1+(k-1) \lambda]^{n} a_{k} z^{k}
$$

Received 20-06-2008, Accepted 18-09-2009.

2000 Mathematics Subject Classification: $30 \mathrm{C} 45$

Key words and Phrases: Univalent function, Distortion theorem, Neighbourhood, Hadamard product 
when $\lambda=1$, get Salagean differential operator [7]. Further, let $T$ denote the subclass of $A$ which consists of functions of the form

$$
f(z)=z-\sum_{k=2}^{\infty} a_{k} z^{k}, a_{k} \geq 0 .
$$

A function $f(z)$ belonging to $A$ is in the class $D_{\lambda}(\alpha, \beta, \xi ; n)$, if and only if

$$
\left|\frac{\left(D^{n} f(z)\right)^{\prime}-1}{2 \xi\left[\left(D^{n} f(z)\right)^{\prime}-\alpha\right]-\left[\left(D^{n} f(z)\right)^{\prime}-1\right]}\right|<\beta
$$

where $0 \leq \alpha<\frac{1}{2} \xi, 0<\beta \leq 1,1 / 2 \leq \xi \leq 1, n \in N \cup\{0\}, z \in U$. Let

$$
T D_{\lambda}(\alpha, \beta, \xi ; n)=T \cap D_{\lambda}(\alpha, \beta, \xi ; n) \text {. }
$$

\section{MAIN RESULTS}

Theorem 1. Let $f$ be defined by (5). Then $f \in T D_{\lambda}(\alpha, \beta, \xi ; n)$ if and only if

$$
\sum_{k=2}^{\infty}[1+(k-1) \lambda]^{n} k[1+\beta(2 \xi-1)] a_{k} \leq 2 \beta \xi(1-\alpha)
$$

$0 \leq \alpha<\frac{1}{2} \xi, 0<\beta \leq 1,1 / 2 \leq \xi \leq 1, n \in N \cup\{0\}, \lambda \geq 0$.

Proof. For $|z|=1$, we get

$$
\begin{aligned}
& \left|\left(D^{n} f(z)\right)^{\prime}-1\right|-\beta\left|2 \xi\left[\left(D^{n} f(z)\right)^{\prime}-\alpha\right]-\left[\left(D^{n} f(z)\right)^{\prime}-1\right]\right| \\
& =\left|-\sum_{k=2}^{\infty}[1+(k-1) \lambda]^{n} k a_{k} z^{k-1}\right| \\
& \quad-\beta\left|2 \xi(1-\alpha)-2 \xi \sum_{k=2}^{\infty}[1+(k-1) \lambda]^{n} k a_{k} z^{k-1}+\sum_{k=2}^{\infty}[1+(k-1) \lambda]^{n} k a_{k} z^{k-1}\right| \\
& \quad \leq \sum_{k=2}^{\infty}[1+(k-1) \lambda]^{n} k[1+\beta(2 \xi-1)] a_{k}-2 \beta \xi(1-\alpha) \\
& \leq 0
\end{aligned}
$$

by hypothesis. Thus by maximum modulus theorem, we have $f \in T D_{\lambda}(\alpha, \beta, \xi ; n)$.

Conversely, suppose that $f \in T D_{\lambda}(\alpha, \beta, \xi ; n)$ hence the condition (6) gives us

$$
\begin{aligned}
& \left|\frac{\left(D^{n} f(z)\right)^{\prime}-1}{2 \xi\left[\left(D^{n} f(z)\right)^{\prime}-\alpha\right]-\left[\left(D^{n} f(z)\right)^{\prime}-1\right]}\right| \\
& =\left|\frac{-\sum_{k=2}^{\infty}[1+(k-1) \lambda]^{n} k a_{k} z^{k-1}}{2 \xi(1-\alpha)-(2 \xi-1) \sum_{k=2}^{\infty}[1+(k-1) \lambda]^{n} k a_{k} z^{k-1}}\right|<\beta .
\end{aligned}
$$


Since $|\operatorname{Re}(z)|<|z|$ for all $z$, we obtain

$$
\operatorname{Re}\left\{\frac{-\sum_{k=2}^{\infty}[1+(k-1) \lambda]^{n} k a_{k} z^{k-1}}{2 \xi(1-\alpha)-(2 \xi-1) \sum_{k=2}^{\infty}[1+(k-1) \lambda]^{n} k a_{k} z^{k-1}}\right\}<\beta .
$$
function

Letting $z \rightarrow 1^{-}$through real values, we get (8). The result is sharp for the

$$
f(z)=z-\frac{2 \beta \xi(1-\alpha)}{[1+(k-1) \lambda]^{n} k[1+\beta(2 \xi-1)]} z^{k}, k \geq 2 .
$$

Corollary 1. Let $f \in T$ belong to the class $T D_{\lambda}(\alpha, \beta, \xi ; n)$ then

$$
a_{k} \leq \frac{2 \beta \xi(1-\alpha)}{[1+(k-1) \lambda]^{n} k[1+\beta(2 \xi-1)]}, k \geq 2
$$

Theorem 2. Let $f \in T$ belong to the class $T D_{\lambda}(\alpha, \beta, \xi ; n)$, then for $|z| \leq r<1$, we have

$$
r-r^{2} \frac{\beta \xi(1-\alpha)}{1+\beta(2 \xi-1)} \leq\left|D^{n} f(z)\right| \leq r+r^{2} \frac{\beta \xi(1-\alpha)}{1+\beta(2 \xi-1)}
$$

and

$$
1-r \frac{2 \beta \xi(1-\alpha)}{1+\beta(2 \xi-1)} \leq\left|\left(D^{n} f(z)\right)^{\prime}\right| \leq 1+r \frac{2 \beta \xi(1-\alpha)}{1+\beta(2 \xi-1)} .
$$

bounds given by (10) and (11) are sharp.

Proof. By Theorem 1, we have

$$
\sum_{k=2}^{\infty}[1+(k-1) \lambda]^{n} k[1+\beta(2 \xi-1)] a_{k} \leq 2 \beta \xi(1-\alpha)
$$

then, we have

$$
2(1+\lambda)^{n}[1+\beta(2 \xi-1)] a_{k} \leq \sum_{k=2}^{\infty}[1+(k-1) \lambda]^{n} k[1+\beta(2 \xi-1)] a_{k} \leq 2 \beta \xi(1-\alpha)
$$

then,

$$
\sum_{k=2}^{\infty} a_{k} \leq \frac{2 \beta \xi(1-\alpha)}{2(1+\lambda)^{n}[1+\beta(2 \xi-1)]}
$$


Hence

$$
\begin{aligned}
\left|D^{n} f(z)\right| & \leq|z|+\sum_{k=2}^{\infty}\left|[1+(k-1) \lambda]^{n} a_{k} z^{k}\right| \\
& \leq|z|+|z|^{2}(1+\lambda)^{n} \sum_{k=2}^{\infty} a_{k} \\
& \leq r+r^{2}(1+\lambda)^{n} \sum_{k=2}^{\infty} a_{k} \\
& \leq r+r^{2} \frac{\beta \xi(1-\alpha)}{1+\beta(2 \xi-1)},
\end{aligned}
$$

and

$$
\begin{aligned}
\left|D^{n} f(z)\right| & \geq|z|-\sum_{k=2}^{\infty}\left|[1+(k-1) \lambda]^{n} a_{k} z^{k}\right| \\
& \geq|z|-|z|^{2}(1+\lambda)^{n} \sum_{k=2}^{\infty} a_{k} \\
& \geq r-r^{2}(1+\lambda)^{n} \sum_{k=2}^{\infty} a_{k} \\
& \geq r-r^{2} \frac{\beta \xi(1-\alpha)}{1+\beta(2 \xi-1)}
\end{aligned}
$$

thus (10) is true. Further,

$$
\begin{aligned}
\left|\left(D^{n} f(z)\right)^{\prime}\right| & \leq 1+2 r(1+\lambda)^{n} \sum_{k=2}^{\infty} a_{k} \\
& \leq 1+r \frac{2 \beta \xi(1-\alpha)}{1+\beta(2 \xi-1)}
\end{aligned}
$$

and

$$
\begin{aligned}
\left|\left(D^{n} f(z)\right)^{\prime}\right| & \geq 1-2 r(1+\lambda)^{n} \sum_{k=2}^{\infty} a_{k} \\
& \geq 1-r \frac{2 \beta \xi(1-\alpha)}{1+\beta(2 \xi-1)} .
\end{aligned}
$$

The result is sharp for the function $f(z)$ defined by

$$
f(z)=z-\frac{2 \beta \xi(1-\alpha)}{1+\beta(2 \xi-1)} z^{2}, z= \pm r .
$$


Theorem 3. Let $n \in N \cup\{0\}, \lambda \geq 0,0 \leq \alpha_{1} \leq \alpha_{2}<\frac{1}{2} \xi, 0<\beta \leq 1,1 / 2 \leq \xi \leq 1$. Then $T D_{\lambda}\left(\alpha_{2}, \beta, \xi ; n\right) \subset T D_{\lambda}\left(\alpha_{1}, \beta, \xi ; n\right)$.

Proof. By assumption we have

$$
\frac{2 \beta \xi\left(1-\alpha_{2}\right)}{[1+(k-1) \lambda]^{n} k[1+\beta(2 \xi-1)]} \leq \frac{2 \beta \xi\left(1-\alpha_{1}\right)}{[1+(k-1) \lambda]^{n} k[1+\beta(2 \xi-1)]}
$$

Thus, $f(z) \in T D_{\lambda}\left(\alpha_{2}, \beta, \xi ; n\right)$ implies that

$$
\sum_{k=2}^{\infty}[1+(k-1) \lambda]^{n} a_{k} \leq \frac{2 \beta \xi\left(1-\alpha_{2}\right)}{k[1+\beta(2 \xi-1)]} \leq \frac{2 \beta \xi\left(1-\alpha_{1}\right)}{k[1+\beta(2 \xi-1)]}
$$

then $f(z) \in T D_{\lambda}\left(\alpha_{1}, \beta, \xi ; n\right)$.

Theorem 4. The set $T D_{\lambda}(\alpha, \beta, \xi ; n)$ is the convex set.

Proof. Let $f_{i}(z)=z-\sum_{k=2}^{\infty} a_{k, i} z^{k}(i=1,2)$ belong to $T D_{\lambda}(\alpha, \beta, \xi ; n)$ and let $g(z)=\zeta_{1} f_{1}(z)+\zeta_{2} f_{1}(z)$ with $\zeta_{1}$ and $\zeta_{2}$ non negative and $\zeta_{1}+\zeta_{2}=1$, we can write

$$
g(z)=z-\sum_{k=2}^{\infty}\left(\zeta_{1} a_{k, 1}+\zeta_{2} a_{k, 2}\right) z^{k}
$$

It is sufficient to show that $g(z)=T D_{\lambda}(\alpha, \beta, \xi ; n)$ that means

$$
\begin{aligned}
\sum_{k=2}^{\infty}[1 & +(k-1) \lambda]^{n} k[1+\beta(2 \xi-1)]\left(\zeta_{1} a_{k, 1}+\zeta_{2} a_{k, 2}\right) \\
& =\zeta_{1} \sum_{k=2}^{\infty}[1+(k-1) \lambda]^{n} k[1+\beta(2 \xi-1)] a_{k, 1}+\zeta_{2} \sum_{k=2}^{\infty}[1+(k-1) \lambda]^{n} k[1+\beta(2 \xi-1)] a_{k, 2} \\
& \leq \zeta_{1}(2 \beta \xi(1-\alpha))+\zeta_{2}(2 \beta \xi(1-\alpha)) \\
& =\left(\zeta_{1}+\zeta_{2}\right)(2 \beta \xi(1-\alpha)) \\
& =2 \beta \xi(1-\alpha)
\end{aligned}
$$

Thus $g(z) \in T D_{\lambda}(\alpha, \beta, \xi ; n)$.

We shall now present a result on extreme points in the following theorem

Theorem 5. Let $f_{1}(z)=z$ and

$$
f(z)=z-\frac{2 \beta \xi(1-\alpha)}{[1+(k-1) \lambda]^{n} k[1+\beta(2 \xi-1)]} z^{k}
$$


for all $k \geq 2, n \in N \cup\{0\}, \lambda \geq 0,0 \leq \alpha<\frac{1}{2} \xi, 0<\beta \leq 1,1 / 2 \leq \xi \leq 1$. Then $f(z)$ is in the subclass $T D_{\lambda}(\alpha, \beta, \xi ; n)$ if and only if it can be expressed in the form $f(z)=\sum_{k=2}^{\infty} \gamma_{k} z^{k}$ where $\gamma_{k} \geq 0$ and $\sum_{k=2}^{\infty} \gamma_{k}=1$ or $1=\gamma_{1}+\sum_{k=2}^{\infty} \gamma_{k}$.

Proof. Let $f(z)=\sum_{k=2}^{\infty} \gamma_{k} z^{k}$ where $\gamma_{k} \geq 0$ and $\sum_{k=2}^{\infty} \gamma_{k}=1$. Thus

$$
f(z)=z-\sum_{k=2}^{\infty} \frac{2 \beta \xi(1-\alpha)}{[1+(k-1) \lambda]^{n} k[1+\beta(2 \xi-1)]} \gamma_{k} z^{k}
$$

and we obtain

$$
\begin{gathered}
\sum_{k=2}^{\infty}\left(\frac{[1+(k-1) \lambda]^{n} k[1+\beta(2 \xi-1)]}{2 \beta \xi(1-\alpha)} \gamma_{k} \times \frac{2 \beta \xi(1-\alpha)}{[1+(k-1) \lambda]^{n} k[1+\beta(2 \xi-1)]}\right) \\
=\sum_{k=2}^{\infty} \gamma_{k}=1-\gamma_{1} \leq 1 .
\end{gathered}
$$

In view of Theorem 1 , this show that $f(z) \in T D_{\lambda}(\alpha, \beta, \xi ; n)$.

Conversely, suppose that of the form (5) belong to $T D_{\lambda}(\alpha, \beta, \xi ; n)$ then

$$
a_{k} \leq \frac{2 \beta \xi(1-\alpha)}{[1+(k-1) \lambda]^{n} k[1+\beta(2 \xi-1)]}, k \geq 2,
$$

Putting

$$
\gamma_{k}=\frac{[1+(k-1) \lambda]^{n} k[1+\beta(2 \xi-1)]}{2 \beta \xi(1-\alpha)}
$$

and $\gamma_{1}=1-\sum_{k=2}^{\infty} \gamma_{k}$, then we have $f(z)=\gamma_{1} f_{1}(z)+\sum_{k=2}^{\infty} \gamma_{k} f_{k}(z)$. This completes the proof.

\section{NEIGHBOURHOOD AND HADAMARD PRODUCT PROPERTIES}

Definition 1. [6] Let $\gamma_{k} \geq 0$ and $f(z) \in T$ of the form (5). The $(k, \gamma)$-neighbourhood of a function $f(z)$ defined by

$$
N_{(k, \gamma)}(f)=\left\{g \in T: g(z)=z-\sum_{k=2}^{\infty} b_{k} z^{k} \text { and } \sum_{k=2}^{\infty} k\left|a_{k}-b_{k}\right| \leq \gamma\right\}
$$

For the identity function $e(z)=z$ we have

$$
N_{(k, \gamma)}(e)=\left\{g \in T: g(z)=z-\sum_{k=2}^{\infty} b_{k} z^{k} \text { and } \sum_{k=2}^{\infty} k\left|b_{k}\right| \leq \gamma\right\} .
$$


Theorem 5. Let

$$
\gamma=\frac{2 \beta \xi(1-\alpha)}{(1+\lambda)^{n}[1+\beta(2 \xi-1)]} .
$$

Then $T D_{\lambda}(\alpha, \beta, \xi ; n) \subset N_{(k, \gamma)}(e)$.

Proof. Let $f \in T D_{\lambda}(\alpha, \beta, \xi ; n)$ then we have

$$
\begin{aligned}
2(1+\lambda)^{n}[1+\beta(2 \xi- & 1)] \sum_{k=2}^{\infty} a_{k} \\
& \leq \sum_{k=2}^{\infty}[1+(k-1) \lambda]^{n} k[1+\beta(2 \xi-1)] a_{k} \\
& \leq 2 \beta \xi(1-\alpha),
\end{aligned}
$$

therefore

$$
\sum_{k=2}^{\infty} a_{k} \leq \frac{\beta \xi(1-\alpha)}{(1+\lambda)^{n}[1+\beta(2 \xi-1)]},
$$

also we have for $|z|<r$

$$
\left|f^{\prime}(z)\right| \leq 1+|z| \sum_{k=2}^{\infty} k a_{k} \leq 1+r \sum_{k=2}^{\infty} k a_{k} .
$$

In view (14), we have

$$
\left|f^{\prime}(z)\right| \leq 1+r \frac{2 \beta \xi(1-\alpha)}{(1+\lambda)^{n}[1+\beta(2 \xi-1)]} .
$$

From above inequalities we get

$$
\sum_{k=2}^{\infty} k a_{k} \leq \frac{2 \beta \xi(1-\alpha)}{(1+\lambda)^{n}[1+\beta(2 \xi-1)]}=\gamma
$$

therefore $f \in N_{(k, \gamma)}(e)$.

Definition 2. The function $f(z)$ defined by (5) is said to be a member of the subclass $T D_{\lambda}(\alpha, \beta, \xi, \zeta ; n)$ if there exits a function $g \in T D_{\lambda}(\alpha, \beta, \xi ; n)$ such that

$$
\left|\frac{f(z)}{g(z)}-1\right| \leq 1-\zeta, z \in U, 0 \leq \zeta<1 .
$$


Theorem 6. Let $g \in T D_{\lambda}(\alpha, \beta, \xi ; n)$ and

$$
\zeta=1-\frac{\gamma}{2} d(\alpha, \beta, \xi ; n) .
$$

Then $N_{(k, \gamma)}(g) \subset T D_{\lambda}(\alpha, \beta, \xi, \zeta ; n)$ where $n \in N \cup\{0\}, \lambda \geq 0,0 \leq \alpha<\frac{1}{2} \xi$, $0<\beta \leq 1,1 / 2 \leq \xi \leq 1,0 \leq \zeta<1$ and

$$
d(\alpha, \beta, \xi ; n)=\frac{(1+\lambda)^{n}[1+\beta(2 \xi-1)]}{(1+\lambda)^{n}[1+\beta(2 \xi-1)]-\beta \xi(1-\alpha)} .
$$

Proof. Let $f \in N_{(k, \gamma)}(g)$ then by (14) we have $\sum_{k=2}^{\infty} k\left|a_{k}-b_{k}\right| \leq \gamma$, then $\sum_{k=2}^{\infty} \mid a_{k}-$ $b_{k} \mid \leq \frac{\gamma}{2}$. Since $g \in T D_{\lambda}(\alpha, \beta, \xi ; n)$ we have

$$
\sum_{k=2}^{\infty} b_{k} \leq \frac{\beta \xi(1-\alpha)}{(1+\lambda)^{n}[1+\beta(2 \xi-1)]}
$$

therefore,

$$
\begin{aligned}
\left|\frac{f(z)}{g(z)}-1\right| & <\frac{\sum_{k=2}^{\infty}\left|a_{k}-b_{k}\right|}{1-\sum_{k=2}^{\infty} b_{k}} \\
& \leq \frac{\gamma}{2}\left(\frac{(1+\lambda)^{n}[1+\beta(2 \xi-1)]}{(1+\lambda)^{n}[1+\beta(2 \xi-1)]-\beta \xi(1-\alpha)}\right) \\
& =\frac{\gamma}{2} d(\alpha, \beta, \xi ; n)=1-\zeta .
\end{aligned}
$$

Then by Definition 2, we get $f \in T D_{\lambda}(\alpha, \beta, \xi, \zeta ; n)$.

Theorem 7. Let $f(z)$ and $g(z) \in T D_{\lambda}\left(\alpha_{1}, \beta, \xi ; n\right)$ be of the form (5) such that $f(z)=z-\sum_{k=2}^{\infty} a_{k} z^{k}$ and $g(z)=z-\sum_{k=2}^{\infty} b_{k} z^{k}$ where $a_{k}, b_{k} \geq 0$. Then the Hadamard product $h(z)$ defined by $h(z)=z-\sum_{k=2}^{\infty} a_{k} b_{k} z^{k}$ is in the subclass $T D_{\lambda}\left(\alpha_{2}, \beta, \xi ; n\right)$ where

$$
\alpha_{2} \leq \frac{[1+(k-1) \lambda]^{n} k[1+\beta(2 \xi-1)]-2 \beta \xi\left(1-\alpha_{1}\right)^{2}}{[1+(k-1) \lambda]^{n} k[1+\beta(2 \xi-1)]} .
$$

Proof. By Theorem 1, we have

$$
\sum_{k=2}^{\infty} \frac{[1+(k-1) \lambda]^{n} k[1+\beta(2 \xi-1)]}{2 \beta \xi\left(1-\alpha_{1}\right)} a_{k} \leq 1
$$

and

$$
\sum_{k=2}^{\infty} \frac{[1+(k-1) \lambda]^{n} k[1+\beta(2 \xi-1)]}{2 \beta \xi\left(1-\alpha_{1}\right)} b_{k} \leq 1
$$


We have only to find the largest $\alpha_{2}$ such that

$$
\sum_{k=2}^{\infty} \frac{[1+(k-1) \lambda]^{n} k[1+\beta(2 \xi-1)]}{2 \beta \xi\left(1-\alpha_{2}\right)} a_{k} b_{k} \leq 1 .
$$

Now, by Cauchy-Schwarz inequality, we obtain

$$
\sum_{k=2}^{\infty} \frac{[1+(k-1) \lambda]^{n} k[1+\beta(2 \xi-1)]}{2 \beta \xi\left(1-\alpha_{1}\right)} \sqrt{a_{k} b_{k}} \leq 1,
$$

we need only to show that

$$
\begin{aligned}
& \frac{[1+(k-1) \lambda]^{n} k[1+\beta(2 \xi-1)]}{2 \beta \xi\left(1-\alpha_{2}\right)} a_{k} b_{k} \\
& \quad \leq \frac{[1+(k-1) \lambda]^{n} k[1+\beta(2 \xi-1)]}{2 \beta \xi\left(1-\alpha_{1}\right)} \sqrt{a_{k} b_{k}},
\end{aligned}
$$

equivalently,

$$
\begin{aligned}
\sqrt{a_{k} b_{k}} & \leq \frac{[1+(k-1) \lambda]^{n} k[1+\beta(2 \xi-1)]}{2 \beta \xi\left(1-\alpha_{1}\right)} \times \frac{2 \beta \xi\left(1-\alpha_{2}\right)}{[1+(k-1) \lambda]^{n} k[1+\beta(2 \xi-1)]} \\
& \leq \frac{1-\alpha_{2}}{1-\alpha_{1}} .
\end{aligned}
$$

But from (18), we have

$$
\sqrt{a_{k} b_{k}} \leq \frac{2 \beta \xi\left(1-\alpha_{1}\right)}{[1+(k-1) \lambda]^{n} k[1+\beta(2 \xi-1)]}
$$

Consequently, we need to prove that

$$
\frac{2 \beta \xi\left(1-\alpha_{1}\right)}{[1+(k-1) \lambda]^{n} k[1+\beta(2 \xi-1)]} \leq \frac{1-\alpha_{2}}{1-\alpha_{1}} .
$$

or equivalently, that

$$
\alpha_{2} \leq \frac{[1+(k-1) \lambda]^{n} k[1+\beta(2 \xi-1)]-2 \beta \xi\left(1-\alpha_{1}\right)^{2}}{[1+(k-1) \lambda]^{n} k[1+\beta(2 \xi-1)]} .
$$

Theorem 8. Let $f \in T D_{\lambda}(\alpha, \beta, \xi ; n)$ be defined by (5) and $c$ any real number with $c>-1$ than the function $G(z)$ defined as $G(z)=\frac{c+1}{z^{c}} \int_{0}^{z} s^{c-1} f(s) d s, c>-1$, also belongs to $T D_{\lambda}(\alpha, \beta, \xi ; n)$. 
Proof. By virtue of $G(z)$ it follows from (5) that

$$
\begin{aligned}
G(z) & =\frac{c+1}{z^{c}} \int_{0}^{z}\left(s^{c}-\sum_{k=2}^{\infty} a_{k} s^{k+c-1}\right) d s \\
& =z-\sum_{k=2}^{\infty}\left(\frac{c+1}{c+k}\right) a_{k} z^{k} .
\end{aligned}
$$

But

$$
\sum_{k=2}^{\infty} \frac{[1+(k-1) \lambda]^{n} k[1+\beta(2 \xi-1)]}{2 \beta \xi(1-\alpha)}\left(\frac{c+1}{c+k}\right) a_{k} \leq 1
$$

since $\left(\frac{c+1}{c+k}\right) \leq 1$ and by Theorem 1 , so the proof is complete.

Theorem 8. Let $f \in T D_{\lambda}(\alpha, \beta, \xi ; n)$ be defined by (5) and

$$
F_{\mu}(z)=(1-\mu) z+\mu \int_{0}^{z} \frac{f(s)}{s} d s \quad(\mu \geq 0, z \in U) .
$$

Then $F_{\mu}(z)$ is also in $T D_{\lambda}(\alpha, \beta, \xi ; n)$ if $0 \leq \mu \leq 2$.

Proof. Let $f$ defined by (5) then

$$
\begin{aligned}
F_{\mu}(z) & =(1-\mu) z+\mu \int_{0}^{z}\left(\frac{s-\sum_{k=2}^{\infty} a_{k} s^{k}}{s}\right) d s \\
& =z-\sum_{k=2}^{\infty} \frac{\mu}{k} a_{k} z^{k} .
\end{aligned}
$$

By Theorem 1 and since $\left(\frac{\mu}{k} \leq 1\right)$ we have

$$
\begin{aligned}
\sum_{k=2}^{\infty} \frac{[1+(k-1) \lambda]^{n} k[1+\beta(2 \xi-1)]}{2 \beta \xi(1-\alpha)}\left(\frac{\mu}{k}\right) a_{k} \\
\leq \sum_{k=2}^{\infty} \frac{[1+(k-1) \lambda]^{n} k[1+\beta(2 \xi-1)]}{2 \beta \xi(1-\alpha)}\left(\frac{\mu}{2}\right) a_{k} \leq 1
\end{aligned}
$$

then $F_{\mu}(z)$ is in $T D_{\lambda}(\alpha, \beta, \xi ; n)$.

\section{REFERENCES}

1. O. Altintas, O. OzKan, And H.M. SRIvastava, "Neighbourhood of a class of analytic functions with negative coefficients", Appl. Math. Lett. 13:3 (2000), 63-67. 
2. P.L. Duren, Univalent functions Grundlehren der Mathematischen wissenchaften, Bd.259, Spinger-Verlag, Berlin, Heidelberg and Tokyo, 1983.

3. G. Murugusundaramoorthy, and H.M. Srivastava, "Neighbourhood of certain classes of analytic functions of complex order", J Inequal. Pure Appl. Math. 5:2 (2004), Art. 24. 8 pp.

4. F.M. Al-Oвоudi, "On univalent functions defined by generalized Salagean operator", IJMMS 27 (2004), 1429-1436.

5. S. Ruscheweyh, "Neighborhoods of univalent functions", Proc. Amer. Math. Soc. 81 (1981), 521-527.

6. S. Ruscheweyh, S. And T Sheil Small, "Hadamard products of Schlicht functions and the polya-Schoenberg conjecture, Comment. Math. Helv. 48 (1973), 119-135. Neighbourhood of univalent functions", Proc. Amer. Math. Soc. 81 (1981), 521-527.

7. G.S. Salagean, Subclasses of univalent functions, Complex-Analysis- fifth Romanian -Finnish Seminar, Part I (Bucharest, (1981)), Lecture Notes in Math, Vol.1013, 362372, Springer Berlin, New-York, 1983.

S. B. Joshi: Department of Mathematics, Walchand College of Engineering, Sangli (M.S), India 416415.

E-mail: joshisb@hotmail.com.

N. D. SAngle: Department of Mathematics, Annasaheb Dange College of Engineering, Ashta, Sangli (M.S), India 416301.

E-mail: navneet_sangle@rediffmail.com. 